\title{
In situ Determination of Surface Tension-to-Shear Viscosity Ratio for Quasiliquid Layers on Ice Crystal Surfaces
}

Ken-ichiro Murata, ${ }^{*}$ Harutoshi Asakawa ${ }^{\dagger}$ Ken Nagashima, Yoshinori Furukawa, and Gen Sazaki Institute of Low Temperature Science, Hokkaido University, N19-W8, Kita-ku, Sapporo 060-0819, Japan (Received 18 September 2015; revised manuscript received 13 November 2015; published 17 December 2015)

We have experimentally determined the surface tension-to-shear viscosity ratio (the so-called characteristic velocity) of quasiliquid layers (QLLs) on ice crystal surfaces from their wetting dynamics. Using an advanced optical microscope, whose resolution reaches the molecular level in the height direction, we directly observed the coalescent process of QLLs and followed the relaxation modes of their contact lines. The relaxation dynamics is known to be governed by the characteristic velocity, which allows us to access the physical properties of QLLs in a noninvasive way. Here we quantitatively demonstrate that QLLs, when completely wetting ices, have a thickness of $9 \pm 3 \mathrm{~nm}$ and an approximately 200 times lower characteristic velocity than bulk water, whereas QLLs, when partially wetting ices, have a velocity that is 20 times lower than the bulk. This indicates that ice crystal surfaces significantly affect the physical properties of QLLs localized near the surfaces at a nanometer scale.

Studies of surface melting (or premelting) have a long history going back to the pioneering prediction by Michael Faraday. In 1859, he first hypothesized that thin water layers, now called quasiliquid layers (QLLs), cover ice crystal surfaces even at a temperature below the melting point [1]. This intriguing phenomenon has attracted considerable attention due to a link to various natural phenomena in our daily life: lubrication on ice surfaces, frost heave by ice columns, the morphological change of snow crystals, and the electrification of thunderclouds [2]. At present, surface melting is known to be not limited only to ice but is common in a wide range of crystalline solids, such as metals, semiconductors, rare gases, and various inorganic, organic, and colloidal systems [3-7]. The significance of surface melting and the resulting quasiliquid layer in condensed matter is therefore hard to overestimate.

However, the underlying mechanism of surface melting is still a matter of debate and is far from completely understood, despite numerous experiments and theories, including numerical simulations, that indicate its existence [8-14]. The major bottleneck is the experimental difficulties in the way of direct and accurate observation of QLLs, whose thicknesses are assumed to be less than tens of nanometer, under well-controlled conditions of temperature and vapor pressure. So far, various sophisticated approaches have been attempted to investigate the physical properties of QLLs, e.g., the thickness, the relaxation time, and their microscopic structure [15]. Unfortunately, however, the results obtained exhibit considerable variation, depending on both experimental methods and researchers (see Table S1 in Ref. [16] for details).

Recently, we directly visualized the QLLs on ice crystal surfaces with molecular resolution in the height direction by using laser confocal microscopy combined with differential interference contrast microscopy (LCM-DIM) [16-19]. Contrary to the conventional wisdom that QLLs statically and homogeneously cover ice surfaces, we demonstrated that the behavior of QLLs is indeed dynamic and spatially heterogeneous, and the QLLs have two distinct wetting morphologies: partial wetting (bulk liquid droplet: BLD) and complete wetting states (thin liquid layer: TLL) on bare ice surfaces. This clearly indicates that conventional spatially averaged techniques, although direct and accurate themselves, are highly uncertain as they do not account for the heterogeneous nature of QLLs. Although laser confocal microscopy combined with spectroscopy (e.g., Raman [20] and Brillouin [21]) would also be a promising way to perform in situ measurements, it is nearly impossible to extract information only about QLLs since their thickness is much less than the resolution limit of an optical microscope. Classically, ice lubrication experiments in quartz capillaries have also been employed to measure the viscosity of the lubricating QLL [22]. However, this kind of measurement, even using modern probe-based techniques, require contact, inevitably disturbing the native state of the QLLs [23].

In this Letter, we present an alternative in situ technique to measure the surface tension-to-shear viscosity ratio (characteristic velocity) of QLLs. We shed light on their wetting dynamics, in particular, the motion of the contact line, which is controlled by the characteristic velocity. We also determine the thickness of the TLL from the spreading dynamics of QLLs. Our approach provides an important clue to understanding not only the nature of QLLs but also, more generally, quasi-two-dimensional fluid flow on the nanoscale [24].

In this study, we employed a confocal system (FV300, Olympus Optical Co. Ltd.) attached to an inverted optical 
microscope (IX70, Olympus Optical Co. Ltd.). To prevent the generation of interference fringes, a super luminescent diode (Amonics Ltd., model ASLD68-050-B-FA, $680 \mathrm{~nm}$ ) was used as a light source. An observation chamber is composed of upper and lower $\mathrm{Cu}$ plates, whose temperatures were separately controlled using Peltier elements. At the center of the upper $\mathrm{Cu}$ plate, a cleaved AgI crystal was attached as an ice nucleating agent. We also prepared other ice crystals on the lower $\mathrm{Cu}$ plate, as a source of water vapor to the sample ice crystals. Separate control of the temperatures of the sample and source ice crystals allows us to adjust the temperature of the sample $(T)$ and vapor pressure $(p)$ independently. Note that $p$ is the partial pressure of $\mathrm{H}_{2} \mathrm{O}$ in a nitrogen environment. The total pressure is the atmospheric pressure. In this study, the observation of QLLs was performed on only basal faces of ice crystals. Further details of the experiments are described in Refs. [16-18].

First, we derive the characteristic velocity of BLDs from the relaxation modes of their contact lines. To observe these, we particularly focused on the process of coalescence of small BLDs with large ones because, for enough large droplets, coalescence with small droplets can be regarded as a weak perturbation of the contact lines [25] (Fig. 1(a) and Video S1 in Supplemental Material [28]). As suggested in our previous study [16], the contact angle $\theta$ of BLDs is extremely small (a few degrees, see below), indicating that a flow inside the wedge of BLDs obeys the lubrication approximation [32], where the flow field is nearly horizontal. Under these conditions, the motion of the contact line is intrinsically dominated by the

(a)

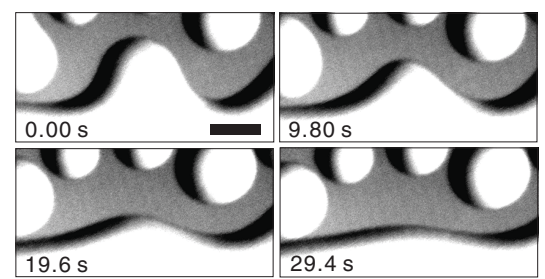

(b)

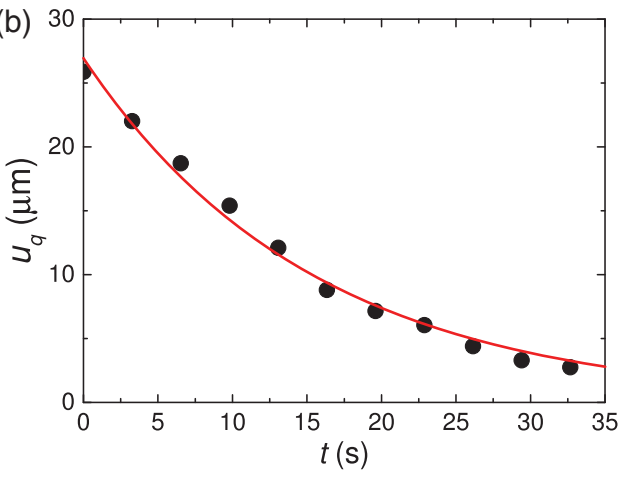

FIG. 1 (color). (a) Pattern evolution of the contact line of the BLD on an ice crystal surface at $T=-0.4^{\circ} \mathrm{C}$ and $p=603.3 \mathrm{~Pa}$. (b) Temporal change of its amplitude $u_{q}$. The data are fitted well by Eq. (1) (the red solid line). The black bar corresponds to $20 \mu \mathrm{m}$. See also Video S1 in the Supplemental Material [28]. balance between (i) the viscous force of the wedge, $F_{V}=3 \ln \theta^{-1} V$, and (ii) the deformation force of the contact line, $F_{E}=-\gamma \theta^{2} q u_{q}$. The relaxation process of the amplitude of contact lines perturbed by a mode with wave vector $q$ is known to be described by the following single exponential decay:

$$
u_{q}=u_{q}(0) \exp \left(-\frac{V^{*} \theta^{3} q}{3 l} t\right)
$$

where $V^{*}=\gamma / \eta$ is the characteristic velocity $(\gamma$ and $\eta$ being the surface tension and shear viscosity of BLDs), $u_{q}(0)$, an initial value of the amplitude. The logarithmic factor $l=\ln (L / a)$ is a cutoff parameter to avoid a singularity at the contact line and at infinite distance, where $a$ is the molecular size ( $3.7 \AA$ for water) and $L$ is approximately the size of the BLDs. From Eq. (1), the relaxation time is given by $\tau_{q}=3 l /\left(V^{*} \theta^{3} q\right)$ [31].

Actually, we can see that in Fig. 1(b) the change in $u_{q}$ is well fitted by Eq. (1), yielding $\tau_{q}=15.5 \pm 0.6 \mathrm{~s}$. The contact angle of BLDs was measured as $\theta=2.0 \pm 0.6^{\circ}$ by means of a two-beam interferometer [16]. Employing $q^{-1} \sim u_{q}(0)=26.9 \pm 0.6 \mu \mathrm{m}$ (from the fitting result) and $l=12.5 \pm 0.1(L=100 \pm 10 \mu \mathrm{m})$ as a set of input parameters, we obtained $V^{*}=2 \pm 1 \mathrm{~m} / \mathrm{s}$. Note that the value of $L$ is the size of a large BLD having an almost flat contact line in Fig. 1(a) (see $t=29.4 \mathrm{~s}$ ). We further analyzed relaxations of other contact lines (see Video S1 in the Supplemental Material [28]) and obtained the average $V^{*}$ as $2 \pm 1 \mathrm{~m} / \mathrm{s}$, the value of which is an order of magnitude smaller than that of bulk water, $42.21 \mathrm{~m} / \mathrm{s}$. Because $\tau_{q}$ includes the $\theta^{3}$ term, leading to a large variation, a good value of $\theta$ is crucial in the precise determination of $V^{*}$. Thus, to obtain a more precise value, further development of our advanced microscopy system, more specifically, the combination of LCM-DIM and a well-controlled interferometer, will be highly required in the future.

Before discussing the $V^{*}$ of TLLs, we focus on the spreading dynamics (a transformation from a BLD to a TLL) in order to evaluate the thickness of TLLs (Fig. 2(a) and Video S2 in the Supplemental Material [28]). The thickness is an essential quantity for the determination of the $V^{*}$ of TLLs, as discussed below. As shown in Fig. 2(b), here we sampled six droplets spreading without coalescence with other QLLs for long periods of time. In Fig. 2(c), we show the time evolution of the radius of QLLs during the spreading at $T=-0.2^{\circ} \mathrm{C}$ and $p=578.9 \mathrm{~Pa}$. The wettability (or spreading coefficient) of QLLs depends on both temperature and vapor pressure. In this case, the transformation was induced by changing the vapor pressure at a constant temperature $\left(T=-0.2^{\circ} \mathrm{C}\right)$. Two dynamic regimes are confirmed in the whole process: in the early stage, the radius slowly increases with time and then more rapidly in the late stage (the purple region). The former 
(a)

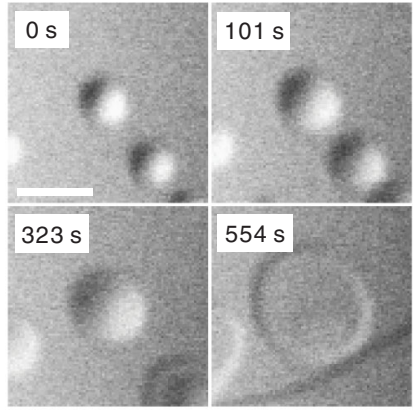

(b)

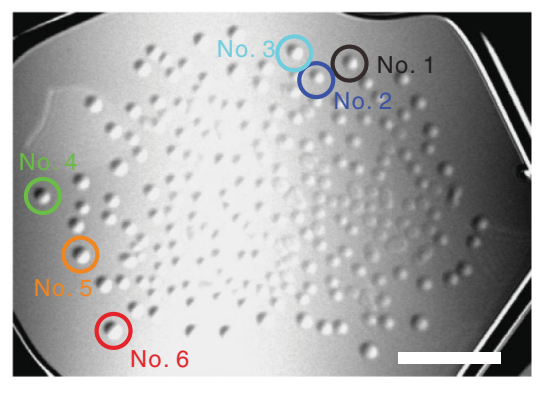

(c)

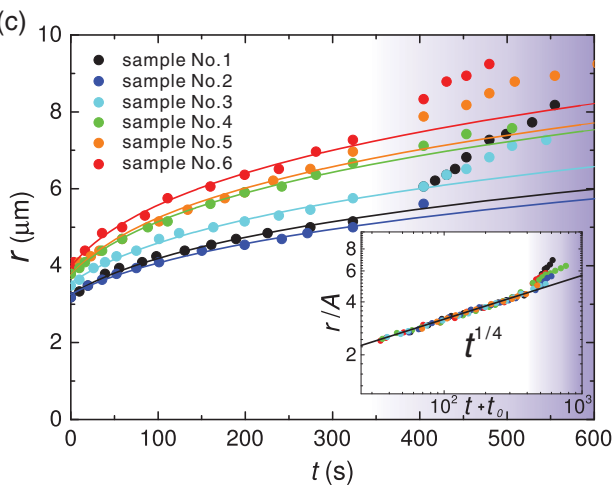

FIG. 2 (color). (a) Spreading dynamics on the ice basal face at $T=-0.2^{\circ} \mathrm{C}$ and $p=578.9 \mathrm{~Pa}$. (b) An initial state $(t=0 \mathrm{~s})$ of the spreading process. We sampled the spreading dynamics of the six droplets indicated by colored circles. The spreading droplet in (a) corresponds to the sample No. 1. (c) Time evolution of the radius of the droplets. We followed the spreading for the six samples in (b). The solid lines represent the best fit of the spreading equation, $r=A\left(t+t_{0}\right)^{1 / 4}$. The purple region indicates the fast growth regime due to supersaturation. The inset indicates the scaled plot of (a) $\left(r / A\right.$ vs $\left.t+t_{0}\right)$. The white bars in (a) and the inset of (b) correspond to $10 \mu$ m and $20 \mu \mathrm{m}$, respectively. See also Video S2 in the Supplemental Material [28].

corresponds to the spreading whereas the latter to the growth of the TLLs by supersaturation.

To analyze the spreading dynamics, we introduced the following power law (see the Supplemental Material [28]), $r=A\left(t+t_{0}\right)^{1 / 4}$, where $A$ is $L(4 S / 3 L l \eta)^{1 / 4}(S$ being the spreading coefficient) and $t_{0}$ is conveniently introduced to represent the initial condition. As shown in the inset of Fig. 2(c), the radial evolution of these six droplets nicely collapses onto a single line by this scaling. Strictly speaking, the whole system in this thermodynamic condition is in a weakly nonequilibrium state (off vapor/ice equilibrium or supersaturation), leading to the growth of both QLLs and ice crystals [33]. In particular, as QLLs decrease their height and approach the TLL state due to spreading, the growth in the direction normal to the surface is limited and consequently the lateral growth is greatly enhanced over that of BLDs capable of isotropic growth. This effect breaks down the scaling relation of the spreading (see the deviation from the single line in the purple region of the inset).

Regarding the BLD as a spherical cap, we can estimate its volume as $V_{b}=(\pi h / 6)\left(3 r_{b}^{2}+h^{2}\right) \sim(\pi / 4) \theta r_{b}^{3}$, where $r_{b}$ and $h$ are the radius and height of the droplets, respectively $\left[h / r_{b} \ll 1\right.$ and $\left.h=r_{b} \tan (\theta / 2) \sim \theta r_{b} / 2\right]$. By contrast, the volume of the TLL is simply written as $V_{t}=\pi r_{t}^{2} e_{m}$ by regarding it as a disk, where $r_{t}$ and $e_{m}$ are the radius and height of the TLL, respectively. From the above scaling, we can access $r_{t}$ without the contribution from the growth by supersaturation. Among the six droplets [Fig. 2(b)], we observed a complete transformation into the TLL state in sample No. 1 (at $t_{c p}=554 \mathrm{~s}$ ) and No. 2 (at $t_{c p}=404 \mathrm{~s}$ ), where $t_{c p}$ is a completion time of the transformation. The value of $r_{t}$ purely coming from the radial change due to spreading is written as $r_{t}=A\left(t_{c p}+t_{0}\right)^{1 / 4}$ and estimated as $5.27 \mu \mathrm{m}$ and $5.89 \mu \mathrm{m}$ for sample No. 1 and No. 2, respectively. Because of volume conservation during the spreading $\left(V_{b}=V_{t}\right)$, we can represent $e_{m}$ as $\theta r_{b}^{3} / 4 r_{t}^{2}$ and consequently obtained the average value of $e_{m}$ as $9 \pm 3 \mathrm{~nm}$.

Interestingly, this thickness agrees well with that of the minimum of the van der Waals potential, estimated by Elbaum and Schick [34] with the Lifshitz theory (3.6 nm). Thus, TLLs can be regarded as the state where QLLs are trapped by this potential minimum whereas the BLDs are QLLs substantially unaffected by this potential, due to $h \gg e_{m}$. Conversely, our results provide experimental evidence for the existence of the local minimum in the effective interfacial potential.

Finally, we evaluate $V^{*}$ of TLLs in the same way as the case of BLDs. Unlike BLDs, however, the hydrodynamic dissipation for TLLs, having a pancake shape, is dominant not near the wedge but inside the body of the fluid. The viscous force for the pancake, moving with $V$, is given by $F_{V}=3 \eta V \zeta / e_{m}[31,35]$, where $\zeta$ is the size of the pancake. In our system, focusing on the contact line perturbation by the absorption, $\zeta$ and $V$ in $F_{V}$ correspond to $u_{q}(0)$ and $d u_{q} / d t$, respectively [36].

In contrast, the precise evaluation of the elastic energy of the contact line is rather difficult owing to the lack of information on the profile of the edges of TLLs. Here, we simply approximated that the contact angle of the edge is $\theta=90^{\circ}$. It is known that the perturbation of the contact line with $q$ influences the surface profile of a sessile droplet in the height direction in the range of $q^{-1}$ [31]. The thickness of TLLs is so thin $\left(e_{m} \ll q^{-1}\right)$ that the profile of the edge cannot recover from the perturbation at $e_{m}$. By properly including this cutoff by $e_{m}$, we obtained the reduced elastic energy as $E=(1 / 4) \gamma q u_{q}^{2}\left(2 q e_{m}\right)$, where the correction term $2 q e_{m}$ corresponds to the cutoff effect. Thus, the deformation force for TLLs is represented as $F_{E}=$ $-\partial E / \partial u_{q}=-\gamma q^{2} u_{q} e_{m}$.

Using the force balance between $F_{V}$ and $F_{E}$ again, we have the following equation: 


$$
u_{q}(t)=u_{q}(0) \exp \left(-\frac{V^{*}}{3 u_{q}(0)}\left(q e_{m}\right)^{2} t\right)
$$

As with BLDs, the contact line relaxation of TLLs has a single exponential form with a relaxation time, $\tau_{q}=3 u_{q}(0) / V^{*}\left(q e_{m}\right)^{2}$. We show in Fig. 3(a), the temporal change of the contact line induced by the coalescence of a small TLL with a large one at $T=-1.6{ }^{\circ} \mathrm{C}$ (see also Video $\mathrm{S} 3$ in the Supplemental Material [28]). Figure 3(b) shows its amplitude to be $u_{q}$. The change in $u_{q}$ is found to be well fitted by Eq. (2). As a result of fitting, we obtained $\tau_{q}=50.7 \pm 1.2 \mathrm{~s}$. Employing $e_{m}=9 \pm 3 \mathrm{~nm}$ and $q^{-1} \sim$ $u_{q}(0)=6.32 \pm 0.09 \mu \mathrm{m}$ (from fitting), we obtained $V^{*}=0.2 \pm 0.1 \mathrm{~m} / \mathrm{s}$. This value is smaller than that of bulk water, $42.21 \mathrm{~m} / \mathrm{s}$. It is worth mentioning that $\gamma_{t}$ (interfacial tension of TLL/air) is almost identical to $\gamma_{b}$ (that of BLD/air) due to the presence of coexisting TLLs and BLDs (see Fig. 5 in Ref. [16]). In this state, the following force balance holds at the contact line among TLLs, BLDs, and vapor: $\gamma_{t}=\gamma_{b} \cos \theta^{\prime} \sim \gamma_{b} \quad\left(\theta^{\prime} \ll 1\right)$, where $\theta^{\prime}$ is a contact angle of BLDs on TLLs. Thus, although the absolute values of the interfacial tension and shear viscosity are unknown, the shear viscosity of TLLs is almost 10 times larger than that of BLDs.

So far, we have ignored water slippage (or a slip length $b$ ) on ices. However, ranging up to tens of nanometers in some cases, $b$ would be of significance not only to hydrodynamics on boundaries [37], but also to wetting dynamics [38]. Here we briefly remark on the validity of

(a)

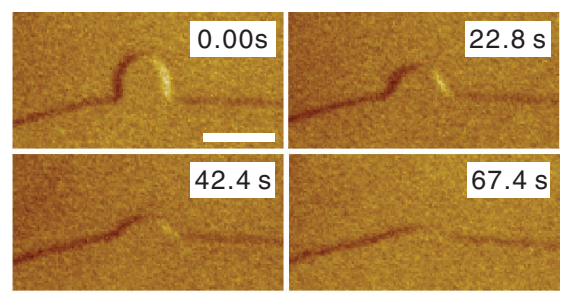

(b)

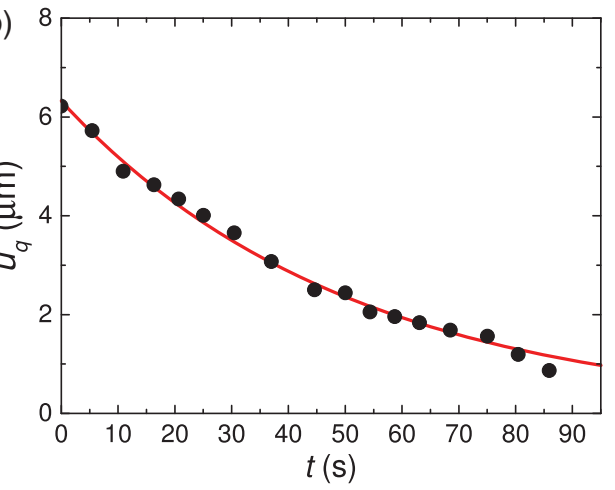

FIG. 3 (color). (a) Pattern evolution of the contact line of the TLL on an ice crystal surface at $T-1.6^{\circ} \mathrm{C}$ and $p=541.2 \mathrm{~Pa}$. (b) Temporal change of its amplitude $u_{q}$. The data are fitted well by Eq. (2) (the red solid line). The white bar corresponds to $10 \mu \mathrm{m}$. See also Video S3 in the Supplemental Material [28]. our assumption $(b=0)$. Recently, Huang et al. [39] demonstrated in their numerical simulations, a simple scaling relation between the contact angle of water droplets and the slip length: $b \propto(1+\cos \theta)^{-2}$, and thus, $b \sim 0$ on hydrophllic surfaces. Hence, $b=0$ in QLLs is true because of $\theta=2.0^{\circ}$ even for BLDs.

In summary, we have quantitatively evaluated the characteristic velocity of QLLs in both the BLD and TLL states from in situ observations of the wetting dynamics of QLLs on ices. We analyzed the relaxation mode of the contact line. We have demonstrated that the characteristic velocity of TLLs is approximately 200 times smaller than that of bulk water while that of BLDs is 20 times smaller than the bulk value. This implies that the closer QLLs are localized on the ice surface, the more strongly the dynamics of QLLs are affected. Furthermore, the spreading dynamics of QLLs tells us that the thickness of TLLs is $9 \pm 3 \mathrm{~nm}$, which is experimental support for the existence of the local minimum in the effective interfacial potential [34].

In this study, we characterized the macroscopic nature of QLLs on the basis of in situ experiments. On the other hand, the microscopic nature (at a molecular scale) and its link to the dynamical modulation by ice surfaces still remain elusive. Recently, although at a metal interface, Limmer et al. numerically demonstrated the slowing down and dynamical heterogeneity of water molecules associated with their structural ordering by water-metal interaction [40], which likely explains the microscopic origin of our results. Moreover, the coarse-grained (local order parameter) approach to ice-water interfaces [14] would provide insight into the dynamic nature of QLLs. In our system, similar experiments on prism and other high-index faces will be a promising way in the next step. The local structure and dynamics of liquid water at and near ice interfaces are supposed to be different, depending on the types of faces of ice crystals, which may affect the value of $V^{*}$ significantly. Novel experimental approaches allowing direct readout of the microscopic properties of QLLs, for example, sum frequency generation microscopy, are also highly desirable in the future.

The authors are grateful to Y. Saito and K. Ishihara (Olympus Engineering Co., Ltd.) for their technical support of LCM-DIM and G. Layton (Northern Arizona University) for the provision of AgI crystals. This work was partially supported by Grants-in-Aid for Scientific Research (A) and for Challenging Exploratory Research (Grants No. 23246001, No. 15H02016, and No. 24656001).

*murata@lowtem.hokudai.ac.jp

†Present address: National Institute of Technology, Anan College, 265 Aoki Minobayashi, Anan, Tokushima 7740017, Japan.

[1] M. Faraday, Experimental Researches in Chemistry and Physics (Taylor and Francis, London, 1859). 
[2] J. G. Dash, A. W. Rempel, and J. S. Wettlaufer, Rev. Mod. Phys. 78, 695 (2006).

[3] T. H. Wee, Y. P. Feng, C. K. Ong, and H. C. Poon, J. Phys. Condens. Matter 8, 6511 (1996).

[4] D. M. Zhu and J. G. Dash, Phys. Rev. Lett. 57, 2959 (1986).

[5] P. Richet, J. Ingrin, B. O. Mysen, P. Courtial, and P. Gillet, Earth Planet. Sci. Lett. 121, 589 (1994).

[6] A. A. Chernov and V. A. Yakovlev, Langmuir 3, 635 (1987).

[7] A. M. Alsayed, M. F. Islam, J. Zhang, P. J. Collings, and A. G. Yodh, Science 309, 1207 (2005).

[8] A. Kouchi, Y. Furukawa, and T. Kuroda, J. Phys. (Paris), Colloq. 48, C1 (1987).

[9] M. Elbaum, S. G. Lipson, and J. G. Dash, J. Cryst. Growth 129, 491 (1993).

[10] X. Wei, P. B. Miranda, and Y. R. Shen, Phys. Rev. Lett. 86, 1554 (2001).

[11] Y. Furukawa and H. Nada, J. Phys. Chem. B 101, 6167 (1997).

[12] M. Conde, C. Vega, and A. Patrykiejew, J. Chem. Phys. 129, 014702 (2008).

[13] M. Watkins, D. Pan, E. Wang, A. Michaelides, J. VandeVondele, and B. Slater, Nat. Mater. 10, 794 (2011).

[14] D. T. Limmer and D. Chandler, J. Chem. Phys. 141, 18C505 (2014).

[15] V. F. Petrenko and R. W. Whitworth, Physics of Ice (Oxford University Press, New York, 1999).

[16] G. Sazaki, S. Zepeda, S. Nakatsubo, M. Yokomine, and Y. Furukawa, Proc. Natl. Acad. Sci. U.S.A. 109, 1052 (2012).

[17] G. Sazaki, S. Zepeda, S. Nakatsubo, E. Yokoyama, and Y. Furukawa, Proc. Natl. Acad. Sci. U.S.A. 107, 19702 (2010).

[18] G. Sazaki, H. Asakawa, K. Nagashima, S. Nakatsubo, and Y. Furukawa, Cryst. Growth Des. 13, 1761 (2013).

[19] H. Asakawa, G. Sazaki, K. Nagashima, S. Nakatsubo, and Y. Furukawa, Cryst. Growth Des. 15, 3339 (2015).

[20] Confocal Raman Microscopy, edited by T. Dieing, O. Hollricher, and J. Toporski (Springer, New York, 2010).

[21] G. Scarcelli and S. H. Yun, Nat. Photonics 2, 39 (2008).

[22] S. S. Barer, N. V. Churaev, B. V. Derjaguin, O. A. Kiseleva, and V. D. Sobolev, J. Colloid Interface Sci. 74, 173 (1980).
[23] H. Bluhm, T. Inoue, and M. Salmeron, Phys. Rev. B 61, 7760 (2000).

[24] S. Granick, Y. Zhu, and H. Lee, Nat. Mater. 2, 221 (2003).

[25] The validity of our analysis, at least in the wavelength regime where gravitational effects can be ignored [26], is supported by the work of Ondarćuhu and Veyssié [27], who constructed an ideal experimental system to analyze relaxation processes of contact lines of relatively thick liquid layers.

[26] Capillarity length, $\kappa^{-1}=\sqrt{\gamma / \rho g}$, of bulk water is around $2 \mathrm{~mm}$, which is smaller than the typical length scale in our experiments $(\leq 100 \mu \mathrm{m})$. Thus, gravitational effects are absent in our system.

[27] T. Ondarćuhu and M. Veyssié, Nature (London) 352, 418 (1991).

[28] See Supplemental Material at http://link.aps.org/ supplemental/10.1103/PhysRevLett.115.256103 for videos showing the wetting dynamics of QLLs and details on the theoretical model of the spreading dynamics, which includes Refs. [29-32].

[29] L. H. Tanner, J. Phys. D 12, 1473 (1979).

[30] P. G. de Gennes, Rev. Mod. Phys. 57, 827 (1985).

[31] P. G. de Gennes, F. Brochard-Wyart, and D. Qéré, Capillarity and Wetting Phenomena: Drops, Bubbles, Pearls, Waves (Springer, New York, 2003).

[32] R. Blossey, Thin Liquid Films (Springer, New York, 2012).

[33] QLLs exist as a steady and metastable state against ice.

[34] M. Elbaum and M. Schick, Phys. Rev. Lett. 66, 1713 (1991).

[35] F. Brochard, Langmuir 5, 432 (1989).

[36] The former replacement means that the internal flow driven by the relaxation of the contact line persists up to the range of the initial amplitude $\left(u_{q}(0)\right)$ with penetration inside the position of the equilibrium contact line.

[37] L. Bocquet and J.-L. Barrat, Soft Matter 3, 685 (2007).

[38] O. Bäumchen, L. Marquant, R. Blossey, A. Münch, B. Wagner, and K. Jacobs, Phys. Rev. Lett. 113, 014501 (2014).

[39] D. M. Huang, C. Sendner, D. Horinek, R. R. Netz, and L. Bocquet, Phys. Rev. Lett. 101, 226101 (2008).

[40] D. T. Limmer, A. P. Willard, P. Madden, and D. Chandler, Proc. Natl. Acad. Sci. U.S.A. 110, 4200 (2013). 


\title{
Supplemental Material: In situ determination of surface tension-to-shear viscosity ratio for quasi-liquid layers on ice crystal surfaces
}

\author{
Ken-ichiro Murata, Harutoshi Asakawa, ${ }^{*}$ Ken Nagashima, Yoshinori Furukawa, and Gen Sazaki \\ Institute of Low Temperature Science, Hokkaido University, N19-W8, Kita-ku, Sapporo 060-0819, Japan
}

\section{THE SPREADING DYNAMICS OF A QUASI-LIQUID LAYER}

It is well-recognized that spreading of non-volatile liquids universally obeys the so-called Tanner's law [1]. In this case, the radial evolution of a sessile droplet (contact angle decay) follows a power law form as $r \propto t^{1 / 10}$ $\left(\theta \propto t^{-3 / 10}\right)$, whose small exponent stems from the presence of a precursor film emerging ahead of the nominal contact line $[2,3]$. For the spreading of QLLs, however, such a precursor film has not been confirmed in the early and intermediate stages even though our advanced microscopy is fully capable of visualizing the film itself. The absence of the precursor film leads to a change in the exponent in Tanner's law. In the followings, we derive a new equation describing the spreading dynamics without the precursor film under the lubrication approximation [4].

We revisit a force, $F$, acting at a contact line. During the spreading, the contact line of the droplet is stretched outward by $F=\gamma_{S V}-\gamma_{S L}-\gamma \cos \theta$, where $\theta$ is the dynamic contact angle during the spreading (Here $\theta \ll$ 1), and $\gamma_{S V}, \gamma_{S L}$ and $\gamma$ are the solid/ gas, solid/liquid and liquid/gas interfacial tensions, respectively. Using the definition of spreading coefficient, $S=\gamma_{S V}-\gamma_{S L}-\gamma$, we can rewrite $F$ as

$$
F=S+\gamma(1-\cos \theta) \sim S+\frac{1}{2} \gamma \theta^{2} .
$$

Spreading means a transformation into the TLL state (a complete wetting state, $S>0$ ). Here due to $\theta \ll 1$, we focus on the case of $F \sim S$. This should balance the viscous force of the wedge, $F_{V}=3 \ln \theta^{-1} V$ ( $V$ being the velocity of the contact line) [3], which gives $V$ as the following relation:

$$
V=\frac{\theta S}{3 l \eta}
$$

The volume of the droplet, $\Omega=(\pi / 4) \theta r^{3}$, is conserved during the spreading. Thus, from the relation of $d \Omega / d t=$
0, we obtain the following differential equation:

$$
\frac{3}{r} \frac{d r}{d t}=-\frac{1}{\theta} \frac{d \theta}{d t} .
$$

Using the relation of $V=d r / d t$ and $r=L \theta^{-1 / 3}$ ( $L$ being $\left.(4 / \pi)^{1 / 3}\right)$, Eq. (3) can be rewritten as

$$
\frac{d \theta}{d t}=-\frac{S}{l L \eta} \theta^{\frac{7}{3}}
$$

Then we obtain as its solution the temporal decay of $\theta$ during the spreading, as

$$
\theta=\left(\frac{4}{3} \frac{S}{l L \eta}\left(t+t_{0}\right)\right)^{-\frac{3}{4}}
$$

where $t_{0}=3 l L \eta / 4 S \theta_{0}^{-4 / 3}$ is determined by the initial condition of the droplet (here $\theta_{0}$ being the contact angle at the start point of the spreading). Furthermore, because of $r=L \theta^{-1 / 3}$, we can easily obtain the radial evolution of the droplet as follows:

$$
r=L\left(\frac{4}{3} \frac{S}{l L \eta}\left(t+t_{0}\right)\right)^{\frac{1}{4}} .
$$

Finally, we note that the precursor film appears in some of the BLDs as TLL states only in the late stage. However, here we do not deal with the dynamics in the late stage due to the complex effects of supersaturation which significantly assists the radial growth of TLLs(see Fig. $2(\mathrm{c}))$.

\footnotetext{
* Present address: National Institute of Technology, Anan College, 265 Aoki Minobayashi, Anan, Tokushima 7740017, Japan

[1] L. H. Tanner, J. Phys. D: Appl. Phys. 12, 1473 (1979).

[2] P. G. de Gennes, Rev. Mod. Phys. 57, 827 (1985).

[3] P. G. de Gennes, F. Brochard-Wyart, and D. Qéré, Capillarity and Wetting Phenomena: Drops, Bubbles, Pearls, Waves (Springer, 2003).

[4] R. Blossey, Thin Liquid Films (Springer, 2012).
} 